\title{
OBITUARI: JOSEP-IGNASI CIRUELO BORGE (1943-2020)
}

\author{
XAVIER ESPLUGA \\ Universitat de Barcelona \\ xespluga@ub.edu \\ ORCID: 0000-0002-6690-1974
}

Enmig de les boires que entenebraren l'annus horribilis de 2020, en el mes de juliol, fou anunciat el traspàs de Josep-Ignasi Ciruelo Borge (Barcelona, 1943-2020), que havia estat professor de Filologia Llatina de la Universitat de Barcelona (UB) (1970-1990) i de la Universidad de Murcia (UM) (1990-1998). A més, Ciruelo va participar, de la mà de Virgilio Bejarano i de Josep Alsina, en les primeres activitats docents de la nova Universitat Autònoma de Barcelona (UAB) (19681970).

Ciruelo s'havia format a la UB (1963-1968) on es va llicenciar en Filologia Clàssica (1968). Successivament, entre 1968 i 1971, gaudí d'una beca de formació del personal docent $\mathrm{i}$ investigador que li serví per cursar els estudis de doctorat en Filologia Clàssica en aquesta mateixa institució. Al cap de poc temps, l'any 1971, defensà la seva tesi doctoral, elaborada sota la direcció de Virgilio Bejarano i dedicada a la figura de Sal·lusti, ${ }^{1}$ autor que treballaria i traduiria més d'una vegada. Els resultats de la seva tesi doctoral foren publicats, l'any 1973, com a monografia independent, a la collecció Convivium de l'Editorial Ariel que aleshores dirigia Josep Alsina. ${ }^{2}$

Pel que fa a la seva participació en les primeres activitats de la UAB, cal tenir present que Frederic Udina, l'encarregat de l'organització de la Facultat de Filosofia i Lletres, havia escollit dues persones per a l'àrea de Filologia Llatina: d'una banda, el ja mencionat Virgilio Bejarano, que feia poc que s'havia incorporat a la UB com a Professor Agregat; de l'altra, Eduard Valentí i Fiol († 1971), aleshores Catedràtic de Llatí de l'Instituto Nacional de Enseñanza Media Jaime Balmes de Barcelona. Consta, doncs, que de la mà de Bejarano i d'Alsina (cridat per Udina per impartir Llengua grega) Ciruelo s'involucrà en aquestes primeres activitats docents de la $U A B$, fins que les directrius ministerials l'obligaren - a ells $\mathrm{i}$ a tots aquells que impartien docència en dos centres universitaris - a triar una única institució.

En qualsevol cas, des del curs acadèmic 1970/1971 i fins al 1974, Ciruelo també ocupà diverses places de professorat dit no numerari - els famosos $P N N-$ a la secció de Filologia Llatina de la UB, places que, d'any en any, variaven de denominació i de sou. ${ }^{3}$ L'any 1974 guanyà el concurs estatal de Professor Adjunt

\footnotetext{
${ }^{1}$ Ciruelo 1971.

2 Ciruelo 1973.

3 A les memòries acadèmiques de la UB trobo les posicions següents: Professor Adjunt (Provisional) (1970-1971) en què va impartir l'assignatura d'Historiografia romana, una de les seves
} 
de Filologia Llatina i prengué possessió de l'Adjuntia corresponent a la UB amb data 1r de setembre de 1975. Arran de la Llei de Reforma Universitària (LRU) de l'agost del 1983, la plaça esmentada es transformà en una titularitat de Filologia Llatina que Ciruelo conservà fins al 1990. El 23 de març d'aquest darrer any esdevingué Catedràtic de Filologia Llatina a la UM, condició que mantingué fins a la seva jubilació el 30 de novembre de 1998, combinant-la amb llargs períodes en comissió de serveis a la Universidad Complutense de Madrid. ${ }^{4}$

En els dos primers anys de vida de la nova UAB a Sant Cugat (1968/1969 i 1969/1970), Ciruelo tornà a coincidir amb Valentí, que havia estat el seu professor de Llatí de Batxillerat al Balmes. Allà mateix també es retrobà amb Pedro-Luis Cano († 2018), deixeble de Valentí, amb qui col-laboraria, més endavant, en més d'una iniciativa, i amb qui compartia la devoció reverencial per la figura del mestre. ${ }^{5}$ De fet, 1 'ombra del llatinista de Pals plana en les primeres col-laboracions de Ciruelo i Cano, en particular en una antologia de textos de prosistes i poetes llatins, ${ }^{6}$ que tots dos van publicar l'any 1972 a l'Editorial Bosch, la casa editorial on Valentí havia anat publicant, des del 1943, les seves conegudes obres de caràcter propedèutic (llibres de text i gramàtiques). La col·laboració entre ambdós amics continuà després que els seus camins professionals es bifurquessin, perquè durant els anys 80 Ciruelo i Cano foren socis en la preparació de material audiovisual relatiu al món clàssic, en una pionera iniciativa de divulgació científica. Així, tots dos són autors del guió del vídeo titulat Aproximación a la Roma clásica, que van produir el 1988 per al Departament de Filologia Clàssica de la Universidad Nacional de Educación a Distancia. ${ }^{7}$

L'esperit didàctic de Ciruelo, heretat de Valentí i compartit amb Cano, s'entreveu en algunes iniciatives del llatinista barceloní, com ara la seva participació en una nova edició de la traducció castellana de la Littérature latine de Jean Bayet, un dels manuals de literatura llatina més emprats a les universitats espanyoles. De fet, la Littérature latine de Bayet havia aparegut en francès el 1965, publicada per Armand Colin; fou traduïda immediatament al castellà, a cura d'Andrés Espinosa Alarcón (deixeble d'Alsina); en efecte, la versió castellana va aparèixer el 1966 a la col·lecció Convivium de l'Editorial Ariel amb un pròleg del mateix Alsina, signat «octubre de 1965» (és a dir, el mateix any en què va sortir a la llum l'original en francès). En aquesta col-lecció Convivium, de 1966 a 1982, la Literatura latina de Bayet tingué sis reimpressions $\left(1966^{1} ; 1970^{2} ; 1972^{3} ; 1975^{4} ; 1_{1981}^{5}\right.$;

especialitats; Professor Agregat Interí (1971-1972); Professor Adjunt per Oposició adscrit a la primera càtedra de Filologia Llatina (1972-1973); Professor Adjunt Interí (1973-1974).

${ }^{4}$ En concret, de 1990 a 1994, estigué a la Complutense, en comissió de serveis, impartint docència al Màster Universitari de Edició (1991-1993), del qual fou també director (1991-1993). En aquests anys, també donà cursos a la Facultad de Ciencias de la Información i fou el director del Servicio de investigación y difusión bibliográfica en soporte micorfilm de la mateixa Universitat madrilenya.

${ }^{5}$ Per a aquestes colllaboracions, vegeu també Espluga 2019.

${ }^{6}$ Ciruelo, Cano 1972.

${ }^{7}$ Ciruelo, Cano 1988. 
$\left.1982^{6}\right)$; en destaca la cinquena, publicada l'any 1981, amb motiu del bimil-lenari de la mort de Virgili, per a la qual Alsina redactà un nou pròleg. Ara bé, l'any 1983 l'Editorial Ariel dugué a terme una nova edició - qualificada com la setena en castellà-, inclosa a la col-lecció Letras e Ideas / Instrumenta, que dirigia Francisco Rico. ${ }^{8}$ Ciruelo assumí la cura d'aquesta renovada edició, a la qual afegí un nou pròleg signat «junio de 1983».9 ${ }^{9}$ 'edició comportava alguns canvis significatius en la presentació del material propedèutic auxiliar perquè incorporava un afegit de «textos ilustrativos en su original latino», que constitueix 1'Apéndice / Textos latinos originales de les pàgines 491-532; també contenia una Guía bibliográfica (a les pàgines 533-535), en paraules del curador, «más escueta y general», pensada perquè l'estudiant hi pogués accedir amb facilitat.

En aquest vessant divulgatiu, Ciruelo col-laborà assíduament amb la Casa Editorial Bosch, si més no des del 1974, continuant, d'aquesta manera, la tasca de Valentí, el qual havia impulsat la publicació de les dues sèries de la Colección de textos clásicos latinos, pensades com a instruments d'aprenentatge a disposició dels estudiants. La primera sèrie incloïa només el text llatí, mentre que la segona aplegava el text llatí, una traducció castellana interlineal i una traducció lliure. En aquesta segona sèrie es recuperaren traduccions antigues (com ara el llibre I de l'Eneida de Lluís Segalà), però també es proposaren versions modernes. Ciruelo hi firmà dos volums: d'una banda, la Conjuración de Catilina, apareguda a la darreria de 1974 (la introducció està firmada «noviembre de 1974»); ${ }^{10}$ de l'altra, el llibre primer de les Metamorfosis d'Ovidi, aparegut el 1976. ${ }^{11}$ Com a complement d'aquesta tasca divulgativa duta a terme en els primers anys de la seva carrera acadèmica, Ciruelo redactà diverses veus per al Diccionario Enciclopédico Salvat Universal (1969-1974) ${ }^{12}$ i per a la Gran Enciclopèdia Catalana (1973-1980). ${ }^{13}$

Ciruelo fou un traductor prolífic - del llatí al castellà i també al català d'autors centrals de la llatinitat clàssica (Plaute, Catul, Sal·lusti, Ovidi). És seva, per exemple, la traducció catalana dels fragments de les Històries de Sal·lusti (i els passatges atribuïts també a l'historiador), que publicà la Fundació Bernat Metge

\footnotetext{
${ }^{8}$ Bayet 1983.

${ }^{9}$ Ciruelo 1983a.

${ }^{10}$ Ciruelo $1974 b$.

${ }^{11}$ Ciruelo $1976 b$.

12 Són seves totes aquestes veus que aparegueren en els toms 6 (Cam-Ceul), 7 (Ceut-Corm), 8 (Corn-Dema), 9 (Demb-Empas) i 11 (Extrañ-Gavin), publicats el 1971: «Cimón», «Claudio I», «Corinto», «Apio Claudio el Ciego», «Cleopatra VII Filopátor», «Clístenes», «cohorte», «colonato», «collegium», «comicios», «Cómmodo, Lucio Aurelio», «Dionisio I de Siracusa», «Domiciano», «Filipo II de Macedonia».

13 Es tracta de veus relatives a autors de la literatura grega i llatina, com ara «Cèsar», «Demòstenes», «Horaci», «Juvenal», «Lucreci», «Nevi», «Persi», «Plini el Jove», «Plini el Vell», «Properci», «Sal·lusti», «Suetoni», «Tàcit», «Tibul» i «Varró». També redactà l'article dedicat a la «Llengua llatina» d'aquesta mateixa enciclopèdia.
} 
(acabada el desembre del 1971, però publicada el 1974). ${ }^{14}$ Aquesta traducció inclö̈a els fragments - cartes i discursos - de les Històries de Sal-lusti (on seguia l'ordenació de l'edició de Maurenbrecher), les dues Cartes a Cèsar vell, atribuïdes a l'historiador d'Amitèrnum, i l'intercanvi d'invectives entre el Pseudo-Sal-lusti i el Pseudo-Ciceró. Destaca també la traducció catalana del Pervigilium Veneris, la seva primera contribució científica, ${ }^{15} \mathrm{i}$, sobretot, la versió de Catul que Ciruelo va realitzar l'any 1982, conjuntament amb Jaume Juan, per a la col·lecció La garba d'Edhasa, una versió que, malgrat haver arribat a la quarantena d'edat, conserva la gràcia i la vivacitat pròpia de la gerdor juvenil. ${ }^{16}$

Ara bé, la iniciativa més destacada en aquest àmbit de la traducció dels clàssics fou la creació de la col-lecció Erasmo Textos bilingües de la Casa Editorial Bosch. Juntament amb Alain Verjat, Ciruelo n'exercí la direcció. Fou el cappare de dues de les sèries: Textos latinos (1975-1996), que arribà als 17 títols, i Textos griegos (1977-1986), amb 6 títols (vegeu Apèndix). A més de la direcció, el llatinista barceloní també hi oferí traduccions de collita pròpia, com el Miles gloriosus de Plaute (1975), que inaugurava tota la col-lecció, ${ }^{17}$ o les dues primeres peces de la trilogia eròtica didàctica d'Ovidi (l'Ars amatoria i els Remedia amoris) (1979). ${ }^{18} \mathrm{~A}$ més, l'any 1976, Ciruelo també va assumir la cura editorial d'una nova edició de la traducció del Lucrecio de Valentí, ${ }^{19}$ partint de la versió castellana del De rerum naturae que el llatinista de Pals havia fet el 1961 per a Alma Mater. ${ }^{20}$ Es tractava d'un homenatge pòstum a la memòria del mestre, tal com declara el mateix Ciruelo en una postil.la de la portada interior del llibre:

La presente obra, homenaje póstumo al humanista Eduard Valentí Fiol, ha sido preparada para la imprenta, y revisada según anotaciones, correcciones y comentarios a la Edición, Introducción, Traducción y Notas de su Lucrecio. De la naturaleza (1961), por José Ignacio Ciruelo Borge, discípulo suyo. ${ }^{21}$

Des de ben aviat, Ciruelo s'involucrà activament en les activitats de la Secció Catalana (en origen, Secció de Barcelona) de la Sociedad Española de Estudios Clásicos, que aleshores dirigia Alsina, amb qui estigué molt unit i amb qui col-laborà tot sovint. De fet, el primer article de Ciruelo, del 1969, el dedicat a la traducció del Peruigilium Veneris, aparegué en el $\triangle \Omega P \Omega I \Sigma \Upsilon N O \Lambda I \Gamma \Omega I$, el volum d'homenatge que els deixebles d'Alsina publicaren aquell mateix any amb motiu del desè aniversari de la promoció de l'hel-lenista de Ripoll a Catedràtic de Filologia

\footnotetext{
${ }^{14}$ Ciruelo 1974a.

${ }^{15}$ Ciruelo 1969.

16 Ciruelo, Juan 1982.

${ }_{17}$ Ciruelo 1975a.

18 Ciruelo 1979.

19 Valentí 1976.

20 Valentí 1961.

21 Valentí 1976.
} 
Grega de la UB. ${ }^{22} \mathrm{Al}$ Segon Simposi d'Estudis Clàssics de la dita Secció, celebrat a Vilanova i la Geltrú del 10 al 17 d'abril de 1970, Ciruelo impartí una ponència sobre la prosa llatina del segle II dC. ${ }^{23}$ Successivament, de la Secció Catalana, Ciruelo en fou sots-president, de 1972 a 1982, quan Josep Alsina n'era el president, i també assumí la presidència mateixa de 1982 a 1985. En aquests anys, impulsà l'organització de dos dels simposis de la Secció. El primer, el Sisè Simposi d'Estudis Clàssics, que commemorava el bimilllenari de la mort de Virgili, fou celebrat a la Universitat de Barcelona de 1'11 al 13 de febrer de 1981. Ciruelo, a banda d'exercir-hi la presidència efectiva, hi impartí una conferència sobre Els herois de Lucreci $i$ Virgili recollida a les actes del simposi. ${ }^{24}$ També presidí el Setè Simposi d'Estudis Clàssics, celebrat a Sitges el 1983 i dedicat als gèneres literaris, on pronuncià el discurs d'obertura. ${ }^{25}$ En el següent simposi, el vuitè, celebrat a Reus l'any 1985, hi tingué una comunicació sobre un vers de la Medea senequiana. ${ }^{26}$

Durant els seus anys a la UB - els més productius de tota la seva carrera acadèmica - fou director de diverses memòries de llicenciatura i de la tesi de doctorat de Jaume Juan. ${ }^{27}$ En aquests treballs, centrats en autors diversos (Catul, Apuleu, la poesia èpica), Ciruelo demostrava el seu interès per les parcel-les centrals de la llatinitat clàssica. ${ }^{28}$

Finalment, en els darrers anys que estigué en actiu, és a dir, durant els primers anys 90, realitzà incursions en el fèrtil camp de la tradició clàssica, amb una nota a un sonet de Petrarca i una anàlisi de les metàfores nàutiques a partir d'una de les composicions dels Basia del poeta holandès Jan Everaerts (Iohannes Secundus) que Olga Gete havia traduït per a la col·lecció Erasmo. ${ }^{29}$

Arran del seu trasllat a Múrcia, Ciruelo es va anar distanciant físicament i també de cor, de les universitats catalanes i, en particular, de la UB. Malgrat tot, el record de Ciruelo - «llatinista de ver» com el va definir en el seu moment Ignasi Mascaró ${ }^{30}$ - anirà lligat a la figura de Sal-lusti, a la promoció de materials propedèutics i de traduccions dels clàssics, en particular de la col-lecció Erasmo,

\footnotetext{
22 Ciruelo 1969.

${ }^{23}$ Ciruelo $1975 b$.

${ }^{24}$ Ciruelo 1983b = Ciruelo 1985a. Cal assenyalar que d'aquestes actes hi ha dues edicions: la primera (de tapes clares) fou publicada l'any 1983 per la Facultat de Filologia de la Universitat de Barcelona (disponible en línia a <http://seec.cat/portfolio-item/actes-del-vie-simposi-1983>) amb el simple títol d'Actes del VIè Simposi d'Estudis Clàssics; la segona, amb tapes verdes, fou publicada pel Servei de Publicacions de la UAB el 1985 i adoptà el títol principal de Studia Virgiliana, mantenint Actes del VI Simposi d'Estudis Clàssics com a subtítol.

${ }_{25}$ Ciruelo $1985 b$.

${ }^{26}$ Ciruelo 1986.

27 Juan 1980.

28 Vegeu Fernández Villamil 1972, Clavo Sebastián 1973, Palao Poveda 1973, Rius Nebot 1984.

29 Respectivament Ciruelo 1992 i Ciruelo 1993.

${ }^{30}$ Mascaró 1982, 28.
} 
una iniciativa que, pel seu afany divulgatiu i pel seu caràcter innovador, anticipa tendències que el girar dels anys ha tornat a posar de moda. ${ }^{*}$

\section{APÈNDIX}

\section{Casa Editorial Bosch: Erasmo, Textos Bilingües}

A partir de 1975, la Casa Editorial Bosch va promoure una col-lecció de textos bilingües, adreçada als estudiants universitaris. Cada volum incloïa el text en llengua original (llatí, grec, alemany, anglès, francès, italià), normalment sense aparat crític, la traducció castellana - acompanyada de notes explanatòries a peu de pàgina - , i una extensa introducció que podia incloure també textos auxiliars, com ara cronologies, esquemes mètrics, mapes, bibliografia.

Els títols de la sèrie Textos latinos (1975-1996) no aparegueren amb periodicitat regular, sinó que es concentraren en determinats anys: ${ }^{31}$ una primera fase abraça els anys compresos entre el 1975 i el 1979; una segona tongada aparegué entre 1983 i 1988 (quan també es produí la reedició de títols apareguts anteriorment) ${ }^{32}$ finalment, el darrer títol constitueix una veritable singularitat, ${ }^{33}$ perquè incorpora excepcionalment aparat crític del text editat, sortí el 1996 (en honor a la veritat, també l'edició del Cançoner de Ripoll de Moralejo incorporava les variants manuscrites sota el text llatí).

La majoria de traductors eren o havien estat professors de la UB (Alcina, Alsina, Anglada, Ciruelo, Conejero, Gete, Juan, Miralles, Rubio, Valero), o si hi havien format (Cano); altres tenien contactes indirectes amb Ciruelo o amb la UB (González Rolán, Otón Sobrino, López i Pociña). Hi ha representants de generacions anteriors, començant per Eduard Valentí, de qui Ciruelo recuperà el Lucrecio d'Alma Mater; s'hi uniren també dos catedràtics d'institut reusencs: Oliveri Nortes, aleshores Catedràtic de Llatí a l'Institut de Reus, que tragué l'Elogi de la follia d'Erasme (i també dues de les biografies suetonianes), i Joaquim Mallafrè, llavors també Catedràtic a l'Institut de Reus, en aquest cas d'Anglès, que girà la Utopia de More (amb el text llatí i el text anglès de l'edició de 1551). No voldria deixar de mencionar la col-laboració de la professora sevillana, María Ángeles Durán Ramas, molt estimada als campus universitaris de Sevilla i Màlaga, que s'encarregà de la Hispaniola de Juan de Maldonado.

\footnotetext{
* Voldria agrair l'amabilitat de Joan Francesc Alcina, Esperança Borrell, Rocío Carande, Joan Carbonell, Concepción Fernández, Marc Mayer, José Martínez Gázquez, José Carlos Miralles, Pere Quetglas i Isabel Velázquez. En particular, una gratitud especial va adreçada a Jaume Juan.

${ }^{31}$ Prenc com a referència la data de publicació. De vegades, pot haver-hi discrepàncies entre l'any del dipòsit legal (normalment anterior) i l'any efectiu de la publicació impresa.

32 En aquest grup s'hi ha d'encabir també la Retòrica d'Herenni d'Alcina, començada feia temps, el procés de publicació de la qual, per motius diversos, s'anà allargant. Vegeu Alcina 1991, 323.

33 Juan 1996.
} 
La colllecció de Textos Latinos no només comprèn autors de la llatinitat clàssica (l'anònima Retòrica per a Herenni, Lactanci, Lucreci, Ovidi, Plaute, Properci, Suetoni, Terenci, Tibul), sinó també obres de la llatinitat medieval, com ara el Cançoner de Ripoll (Moralejo), el Pàmfil o l'art d'estimar (Rubio i González Rolán) o el dit Liber Maiolichinus (Juan). ${ }^{34}$ Sorprèn agradablement que s'hi incloguessin obres de l'humanisme neollatí, una parcel-la tradicionalment absent dels plans d'estudi i dels projectes de recerca de la UB. Així, la col-lecció Erasmo aixoplugà les ja mencionades Utopia de More (Mallafrè), l'Elogi de la follia d'Erasme (Nortes), l'obra poètica de Jan Everaerts (Iohannes Secundus) (Gete) i La Española de Juan de Maldonado (Durán Ramas).

Pel que fa a la sèrie de Textos Griegos, de menor entitat, la col·lecció Erasmo comptà amb la colllaboració de professors barcelonins (Alsina, Conejero, Miralles, Valero) i també d'Antonio López Eire que poc abans havia estat Professor Agregat de Filologia Grega a la UAB. Incloïa autors clàssics (Demòstenes, Èsquil, Eurípides), dos textos bizantins -el Poema e historia de Belisario i el Digenís Akritas - a càrrec de Juan Valero, i un tractat d'estètica prou important, el Del sublim del Pseudo-Longí (Alsina), del qual dissortadament no tenim encara la necessària traducció catalana.

\section{Erasmo Textos Latinos (1975-1996)}

1975

CIRUELO BORGE (1975): Plauto. El militar fanfarrón. Introducción, cronología, traducción y notas de José-Ignacio Ciruelo, Barcelona, Bosch (Erasmo Textos Bilingües / Textos Latinos). [reimpr. (1983); segona edició (1998)].

1976

NORTES VAlls (1976): D. Erasmo de Rotterdam. Elogio de la locura. Introducción, nueva traducción y notas de Oliveri Nortes Valls, Barcelona, Bosch (Erasmo Textos Bilingües / Textos Latinos).

VAlentí Fiol (1976): T. Lucrecio Caro. De la naturaleza. Eduard Valentí Fiol, Barcelona, Bosch (Erasmo Textos bilingües / Textos Latinos). ${ }^{35}$ [reimpr. (1985); (1987); (1993)].

\footnotetext{
${ }^{34}$ En la seva edició, Jaume Juan donava a aquesta obra un títol nou, De bello Maioricano libri octo, i l'atribuïa a Laurentius Veronensis (seguint una vella tradició d'estudis). Per a una segona edició crítica, vegeu Juan 2014. Recordo també que aquest text fou objecte de la tesi doctoral de Jaume Vidal Alcover (Vidal Alcover 1976A), de la qual només en fou publicat un extracte (Vidal Alcover 1976B). Per a una traducció catalana, vegeu Mulet Mas 1991. L'edició crítica més recent, amb traducció italiana, és Scalia, Bartola, Guardo 2017.

${ }^{35}$ La primera edició, del 1976, sortí en dos toms; la reimpressió successiva, de 1985, en un únic volum.
} 
1977

MALlAFRÈ GAVALDÀ (1977): Thomas More. Utopía. Introducción, cronología, bibliografía, notas y traducción inédita de Joaquim Mallafrè Gavaldà, Barcelona, Bosch (Erasmo Textos Bilingües / Textos Latinos).

PociÑA, LóPEZ (1977): P. Terencio Afro. El Eunuco. Introducción, cronología, traducción inédita y notas de Andrés Pociña y Aurora López, Barcelona, Bosch (Erasmo Textos bilingües / Textos Latinos).

Rubio, GonzÁlez Rolán (1977): Anónimo. Pánfilo o el arte de amar. Texto, Introducción, traducción, aparato crítico y notas de L. Rubio y T. González Rolán, Barcelona, Bosch (Erasmo Textos Bilingües / Textos Latinos).

1979

CIRUElo BORGE (19791; 19902): Ovidio. Arte de amar. Introducción, cronología, bibliografía, notas y traducción de José-Ignacio Ciruelo. [Remedios del amor], Barcelona, Bosch (Erasmo Textos bilingües / Textos Latinos). ${ }^{36}$ [reimpr. (1983); (1987); (1988)].

GETE CARPIO (1979): Juan Segundo, Besos y otros poemas. Introducción, cronología, bibliografía, notas y traducción de Olga Gete Carpio, Barcelona, Bosch (Erasmo Textos Bilingües / Textos Latinos).

OTÓN SOBRINO (1979): Tibulo. Poemas. Introducción, notas y traducción de Enrique Otón Sobrino, Barcelona, Bosch (Erasmo Textos bilingües / Textos Latinos).

1983

ANGLADA ANFruns (1983): El mito del Ave Fénix. Introducción, textos, traducción y notas de Ángel Anglada Anfruns, Barcelona, Bosch (Erasmo Textos Bilingües / Textos Latinos).

DURÁN RAMAS (1983): Juan de Maldonado. La Española. Edición, introducción, traducción y notas de María Ángeles Durán Ramas, Barcelona, Bosch (Erasmo Textos Bilingües / Textos Latinos).

NORTES VALLS (1983): Suetonio. Tiberio. Calígula. Introducción, cronología, bibliografía, notas y traducción de Oliveri Nortes Valls, Barcelona, Bosch (Erasmo Textos Bilingües / Textos Latinos).

\section{5}

CANO (1985): Propercio. Poemas. Introducción, cronología, bibliografía, notas y traducción de Pedro-Luis Cano Alonso, Barcelona, Bosch (Erasmo Textos bilingües / Textos Latinos).

\section{6}

MORAlEJO (1986): (Anónimo). Cancionero de Ripoll. Texto, traducción, introducción y notas de José-Luis Moralejo, Barcelona, Bosch (Erasmo Textos bilingües / Textos Latinos).

${ }^{36}$ El volum inclou també els Remedia amoris la menció dels quals apareixen a la portada exterior, però no pas a la portada interior, d'on s'extreuen les dades bibliogràfiques. 


\section{8}

JUAN (1988): Horacio. Odas. Introducción, cronología, bibliografía, notas y traducción de Jaume Juan Castelló, Barcelona, Bosch (Erasmo Textos bilingües / Textos Latinos).

\section{1}

AlcinA (1991): [Cicerón]. Retórica a Herenio. Traducción, introducción y notas de Juan Francisco Alcina, Barcelona, Bosch (Erasmo Textos bilingües / Textos Latinos).

\section{6}

JUAN (1996): Lorenzo Veronés. La guerra de Mallorca en ocho libros. Cronología, introducción, edición crítica e indice de Jaume Juan Castelló, Barcelona, Bosch (Erasmo Textos Bilingües / Textos Latinos).

\section{Erasmo Textos Griegos (1977-1983)}

\section{7}

LÓPEZ EIRE (1977): Aristófanes. Las asambleístas. Texto, introducción, nueva traducción y notas de Antonio López Eire, Barcelona, Bosch (Erasmo Textos bilingües / Textos Griegos). MiRALLES (1977): Eurípides. Hipólito. Texto, introducción, nueva traducción y notas de Carlos Miralles, Barcelona, Bosch (Erasmo Textos bilingües / Textos Griegos).

\section{9}

Alsina (1979): Esquilo. La Orestia. Introducción, texto, traducción y notas de José Alsina, Barcelona, Bosch (Erasmo Textos bilingües / Textos Griegos). [reimpr. (1987)]

1980

CONEJERO (1980): Demóstenes. Filípicas. Introducción, traducción y notas de Valentín Conejero Ciriza, Barcelona, Bosch (Erasmo Textos bilingües / Textos Griegos).

\section{1}

VALERO (1981): Basilio Digenís Akritas. Introducción, cronología, bibliografía, notas y traducción inédita de Juan Valero Garrido, Barcelona, Bosch (Erasmo Textos bilingües / Textos Griegos).

\section{3}

VALERO (1983): Poema e historia de Belisario. Introducción, traducción y notas de Juan Valero Garrido, Barcelona, Editorial Bosch (Erasmo Textos bilingües / Textos Griegos).

1996

Alsina (1996): Anónimo. Sobre lo sublime. Aristóteles. Poética. Texto, Introducción, Traducción y Notas de José Alsina Clota, Barcelona, Editorial Bosch (Erasmo Textos bilingües / Textos Griegos). 


\section{BIBLIOGRAFIA}

AlciNA, J. F. (1998), «Salvador Núñez, Retórica a Herenio. Introducción, traducción y notas, Madrid, Gredos, 1997», Cuadernos de Filología Clásica 14, 323-325.

BAYET, J. (1983), Literatura latina. Nueva edición corregida y aumentada con textos latinos, Barcelona, Editorial Ariel (Col. Letras e Ideas).

Ciruelo, J.-I. (1969), «Una versió del Pervigilium Veneris», $\triangle \Omega P \Omega I \Sigma \Upsilon N$ O $\Lambda I \Gamma \Omega I$. Homenatge a Josep Alsina dels seus deixebles, en el desè aniversari de la seva càtedra a la Universitat de Barcelona, Barcelona, Edicions Ariel, 175-187.

Ciruelo, J.-I. (1971), Salustio. Política e historiografía. Memoria para la obtención del grado de doctor (ponente: Virgilio Bejarano Sánchez), Barcelona, Universitat de Barcelona.

Ciruelo, J.-I. (1973), Salustio. Política e historiografía, Barcelona, Ariel (Convivium, 15).

Ciruelo, J.-I. (1974a), G. Sal.lusti Crisp. Apèndix (fragments i obres espúries). Text revisat $i$ traducció de Josep-Ignasi Ciruelo, Barcelona, Fundació Bernat Metge (Escriptors llatins, 187).

CIRUelo, J.-I. (1974b), Salustio. Conjuración de Catilina. Texto, traducción, ordenación directa y versión interlineal por José-Ignacio Ciruelo, Barcelona, Editorial Bosch (Colección de textos clásicos latinos) [reimpr. (1981); (1987)].

CirUelo, J.-I. (1975a $\left.{ }^{1} ; 1998^{2}\right)$, Plauto. El militar fanfarrón. Introducción, cronología, traducción y notas de José-Ignacio Ciruelo, Barcelona, Editorial Bosch (Colección Erasmo Textos Bilingües).

Ciruelo, J.-I. (1975b), «La prosa en Roma durante el siglo II», Roma en el siglo II. Trabajos de la Sección Latina del II Simposio de la Sociedad Española de Estudios Clásicos. Sección de Barcelona, Barcelona, Ediciones de la Universidad de Barcelona, 135-140.

CIRUElo, J.-I. (1976a), «Introducción» a Lucrecio. De la naturaleza. Introducción de JoséIgnacio Ciruelo Borge. Traducción y notas de Eduard Valentí Fiol, Barcelona, Editorial Bosch (Colección Erasmo Textos Bilingües).

Ciruelo, J.-I. (1976b), P. Ovidio Nasón. Metamorfosis. Libro I. Traducción de José-Ignacio Ciruelo Borge, Barcelona, Editorial Bosch (Colección de textos clásicos latinos) [reimpr. (1986)].

Ciruelo, J.-I. (1979), Ovidio. Arte de amar. [Remedios de amor]. Introducción, cronología, bibliografía, notas y traducción de José-Ignacio Ciruelo, Barcelona, Editorial Bosch (Colección Erasmo Textos Bilingües).

Ciruelo, J.-I. (1983a), «Prólogo a la séptima edición castellana», a Bayet (1983), 7.

Ciruelo, J.-I. (1983b), «Els herois de Lucreci i Virgili», Actes del VI Simposi d'Estudis Clàssics, 11-13 de febrer, Barcelona, Secció Catalana de la SEEC - Facultat de Filologia de la Universitat de Barcelona, 21-30 = CIRUELO, J.-I. (1985a), «Els herois de Lucreci i Virgili», Studia Virgiliana, Bellaterra, Secció Catalana de la SEEC - Servei de Publicacions de la Universitat Autònoma de Barcelona, 21-30 . 
Ciruelo, J.-I. (1985b), «Discurs d'obertura», El teatre grec i romà. Actes del VIII Simposi d'Estudis Clàssics de la Secció Catalana de la Sociedad. Española de Estudios Clásicos (Reus, 23-25 abril 1985), Barcelona, Secció Catalana de la SEEC, I-IV.

Ciruelo, J.-I. (1986), «Medea superest (Sen. Medea, v. 166)», Actes del VIII $e^{\grave{S}}$ Simposi d'Estudis Clàssics, Reus 1985, Barcelona, Secció Catalana de la SEEC 1986, 61-64.

Ciruelo, J.-I. (1988), Ovidio. Arte de amar. Remedios de amor, Barcelona, Icaria (Icaria literaria, 32).

CIRUelo, J.-I. (1992), «Nota al soneto XCII del 'Canzioniere' de Petrarca», Humanitas. In honorem Antonio Fontán, Madrid, Gredos, 1992, 475-479.

Ciruelo, J.-I. (1993), «Ioannis Secundi, Basia I, 9-12 (Nota para la historiografía literaria de la metáfora náutica)», en Humanismo y pervivencia del mundo clásico : actas del I Simposio sobre Humanismo y pervivencia del mundo clásico (Alcañiz, 8 al 11 de mayo de 1990), vol. I, Maestre Maestre, J. M. y Pascual Barea, J. (coords.), Alcañiz, Instituto de Estudios Turolenses-Servicio de Publicaciones de la Universidad de Cádiz, 323-328.

Ciruelo, J.-I. I CANO, P.-L. (1972), Textos latinos de prosistas y poetas. Estudios de sintaxis, Barcelona, Editorial Bosch.

Ciruelo, J.-I. I CANO, P.-L. (1988), Aproximación a la Roma clásica. Una producción del Centro de Diseño y Producción de Medios Audiovisuales para el Departamento de Filología Clásica de la UNED. Guión de José-Ignacio Ciruelo, Pedro L. Cano. Realización de Pedro L. Cano, Pedro Valle [DVD], Madrid, UNED.

Ciruelo, J.-I. I JuAN, J. (1982), Catul. Poemes. Edició bilingüe integra. Traducció i notes de Josep Ignasi Ciruelo i Jaume Juan, Barcelona, Edhasa (La Garba, 2).

Clavo Sebastián, M. T. (1973), La cualificacion personal en Catulo. Memoria de licenciatura que presenta María Teresa Clavo Sebastián realizada bajo la dirección del Dr. J.-I. Ciruelo Borge, Barcelona, Universitat de Barcelona.

EsplugA, X. (2019), «Obituari. Pedro Luis Cano (†2018)», Anuari de Filologia. Antiqua et Mediaevalia», 9.1, 92-96.

FERNÁNDEZ VILLAMIL, M. E. (1972), El mito de Io y la metamorfosis de Lucio en Apuleyo. Tesis de licenciatura en Filosofía y Letras (especialidad en Filología Clásica Latina) dirigida por J.-I. Ciruelo, Barcelona, Universitat de Barcelona.

JUAN, J. (1980), La puntuació llatina i la lectura en veu alta. Tesi per a l'obtenció del grau de doctor, dirigida per J.-I. Ciruelo, Barcelona, Universitat de Barcelona.

JUAN, J. (2014): Laurentius Veronensis. De bello Maioricano libri octo iterum edidit Jaume Juan Castelló [en línia], Barcelona, Dipòsit digital UB. Disponible al: $<$ http://diposit.ub.edu/dspace/bitstream/2445/59444/1/de_bello_Maioricano.pdf>.

MASCARÓ, I. (1982), «Joan Ramis. Elegia», Latitud 39. Revista de literatura, llengua i cultura, 12, 28-29.

Mulet MAS, M. (1991), Liber Maiolichinus de gestis Pisanorum illustribus. Traducció de Mireia Mulet Mas, Palma de Mallorca, Societat Arqueològica Lul·liana.

Palao PovedA, M. D. (1973), Sobre los poemas XI y LI de Catulo. Memoria de Licenciatura. Director J.-I. Ciruelo, Barcelona, Universitat de Barcelona. 
RiUs NebOT, E. (1984), Els vents en la poesia èpica llatina: Virgili, Sili Itàlic i Valeri Flac. Memòria de llicenciatura dirigida pel professor J.-I. Ciruelo, Barcelona, Universitat de Barcelona.

ScAliA, G., BARTOlA, A. I GUARDO, M. (2017), Liber Maiorichinus de gestis Pisanorum illustribus. Introduzione e testo critico di Giuseppe Scalia. Commento di Alberto Bartola. Traduzione di Marco Guardo (Edizione Nazionale dei Testi Mediolatini d'Italia, 44 Serie II, 20), Firenze, SISMEL-Edizioni del Galluzzo.

VAlentí FIOL, E. (1961), T. Lucrecio Caro. De la naturaleza. Texto revisado y traducido por Eduardo Valentí, Barcelona, Ediciones Alma Mater (Colección Hispánica de autores griegos y latinos patrocinada por las universidades españolas).

VAlENTí FIOL, E. (1976), T. Lucrecio Caro. De la naturaleza. Eduard Valentí Fiol, Barcelona, Bosch (Erasmo Textos bilingües / Textos Latinos).

VIDAl Alcover, J. (1976A), El llibre de Mallorca. Liber Maiorchinus. Text, traducció, notes i introducció. Tesi doctoral (Director Dr. Joan Bastardas), Barcelona, Universitat de Barcelona, Facultat de Filosofia i Lletres, Secció de Filologia Romànica.

VidAl Alcover, J. (1976B), El Llibre de Mallorca (Liber Maiolichinus o Maiorchinus). Introducció a l'edició del poema llatí i a la seva traducció catalana, Barcelona, Fundació Jaume I. 